\title{
BMJ Open Why does healthcare utilisation differ between socioeconomic groups in OECD countries with universal healthcare coverage? A protocol for a systematic review
}

\author{
Iris Meulman (D) , ${ }^{1,2}$ Ellen Uiters, ${ }^{3}$ Johan Polder, ${ }^{1,2}$ Niek Stadhouders ${ }^{1}$
}

To cite: Meulman I, Uiters E, Polder J, et al. Why does healthcare utilisation differ between socioeconomic groups in OECD countries with universal healthcare coverage? A protocol for a systematic review. BMJ Open 2021;11:e054806. doi:10.1136/ bmjopen-2021-054806

- Prepublication history and additional supplemental material for this paper are available online. To view these files, please visit the journal online (http://dx.doi.org/10.1136/ bmjopen-2021-054806)

Received 23 June 2021 Accepted 27 October 2021

Check for updates

(c) Author(s) (or their employer(s)) 2021. Re-use permitted under CC BY-NC. No commercial re-use. See rights and permissions. Published by BMJ.

${ }^{1}$ Centre for Health and Society, National Institute for Public Health and the Environment, Bilthoven, The Netherlands

${ }^{2}$ Tilburg School of Social and Behavioral Sciences, Tilburg University, Tilburg, The Netherlands

${ }^{3}$ Center for Nutrition, Prevention and Health Services, National Institute for Public Health and the Environment, Bilthoven, The Netherlands

Correspondence to

Iris Meulman;

iris.meulman@rivm.nl

\section{ABSTRACT}

Introduction Even in advanced economies with universal healthcare coverage (UHC), a social gradient in healthcare utilisation has been reported. Many individual, community and healthcare system factors have been considered that may be associated with the variation in healthcare utilisation between socioeconomic groups. Nevertheless, relatively little is known about the complex interaction and relative contribution of these factors to socioeconomic differences in healthcare utilisation. In order to improve understanding of why utilisation patterns differ by socioeconomic status (SES), the proposed systematic review will explore the main mechanisms that have been examined in quantitative research.

Methods and analysis The systematic review will follow the Preferred Reporting Items for Systematic Review and Meta-Analyses guidelines and will be conducted in Embase, PubMed, Scopus, Web of Science, Econlit and Psyclnfo. Articles examining factors associated with the differences in primary and specialised healthcare utilisation between socioeconomic groups in Organisation for Economic Co-operation and Development (OECD) countries with UHC will be included. Further restrictions concern specifications of outcome measures, factors of interest, study design, population, language and type of publication. Data will be numerically summarised, narratively synthesised and thematically discussed. The factors will be categorised according to existing frameworks for barriers to healthcare access.

Ethics and dissemination No primary data will be collected. No ethics approval is required. We intend to publish a scientific article in an international peer-reviewed journal.

\section{INTRODUCTION}

Differences in healthcare utilisation by socioeconomic status (SES) have been widely reported, also in countries with universal healthcare coverage (UHC). ${ }^{1-4}$ Individuals with lower SES generally use more care and have higher healthcare spending. This finding holds for different socioeconomic
Strengths and limitations of this study

- The systematic review will provide a comprehensive overview of individual, community and healthcare system-level factors examined in quantitative research that may contribute to socioeconomic differences in healthcare utilisation.

- Socioeconomic status will be defined by a broad range of indicators beyond commonly applied individual-level characteristics such as educational level or income.

- In data synthesis, a distinction will be made by type of health service and between results that are, in all probability, generalisable and those that are bounded to a specific context.

- The expected heterogeneity in study characteristics and outcome measures of the included studies may limit the comparability and statistical analysis.

indicators (eg, educational attainment, household income or occupational status) and most types of care. ${ }^{4-7}$ Exemptions are reported for physiotherapy, highly specialised care and dental care, which may be used more by higher SES groups. ${ }^{8}$

Levesque et $a l^{9}$ identified barriers and determinants which play a role in different stages in the process of obtaining access to care. Their model consists of five dimensions of accessibility (approachability, acceptability, availability and accommodation, affordability and appropriateness) and five abilities required to obtain access to care (ability to perceive, ability to seek, ability to reach, ability to pay and ability to engage). These interacting dimensions and abilities comprise factors at the individual, community and healthcare system levels. Because healthcare utilisation is often referred to as realised access, ${ }^{9}$ this 
framework is relevant to study factors related to the variation in healthcare utilisation.

Several factors related to socioeconomic differences in healthcare utilisation have been examined. Foremost, a large part of the variation in healthcare utilisation between socioeconomic groups can be explained by differences in health. ${ }^{1451011}$ In addition, other factors, such as age, ${ }^{12}$ health literacy, ${ }^{13}$ lifestyle $^{14}$ or copayments, ${ }^{15}$ are considered to be associated with the variation in healthcare utilisation between socioeconomic groups. Although many factors have been examined separately, a comprehensive overview is missing and little is known about the interrelationship and relative contributions of these factors to socioeconomic differences in healthcare utilisation. In the perspective of the increasing healthcare expenditure, comprehensive knowledge regarding these underlying mechanisms may be particularly relevant for policy-makers.

The proposed review aims to improve understanding of why utilisation patterns differ between socioeconomic groups in Organisation for Economic Co-operation and Development (OECD) countries with UHC. A broad literature study will be conducted to answer the research question "what are the main factors that have been examined in quantitative research for association with the differences in healthcare utilization between socioeconomic groups?'. We focus on primary and specialised healthcare, including general practitioner (GP) care, medical care, hospital care, ambulatory care, mental healthcare and dental care.

\section{METHODS AND STUDY DESIGN}

This systematic review will follow the guidelines proposed by the Preferred Reporting Items for Systematic Review and Meta-Analysis Protocol (PRISMA-P). ${ }^{16} 17$

\section{Eligibility criteria}

Articles meeting the criteria outlined in table 1 will be included. Articles are screened based on the outcome measure, factors of interest, study design, population, language and type of publication. Most importantly, studies are required to (1) have healthcare utilisation as an outcome measure, (2) analyse the difference in healthcare utilisation with respect to SES and (3) examine one or more underlying factors of the difference in healthcare utilisation between socioeconomic groups.

\section{Factors and outcome measures}

Given the focus on healthcare utilisation, studies with health status, mortality, quality of care or waiting times as an outcome measure are not eligible for inclusion. Healthcare utilisation will be divided into different types of care such as GP care, medical care, hospital care, ambulatory care, mental healthcare and dental care. Prevention is excluded because the underlying reasons

Table 1 Eligibility criteria

\begin{tabular}{|c|c|c|}
\hline & Inclusion criteria & Exclusion criteria \\
\hline Outcome measure & $\begin{array}{l}\text { Difference in healthcare utilisation between } \\
\text { socioeconomic groups } \\
\text { Primary and specialised types of healthcare } \\
\text { Utilisation is expressed as volume, cost/ } \\
\text { expenses or access to care } \\
\text { Utilisation is analysed with respect to SES } \\
\text { SES is expressed as broad measures of } \\
\text { income, education, occupational status, a } \\
\text { composite measure, or any other indicator } \\
\text { used as a proxy for SES }\end{array}$ & $\begin{array}{l}\text { No measure of socioeconomic inequality } \\
\text { Preventive care or screening } \\
\text { Utilisation is expressed as the quality of care or } \\
\text { waiting times } \\
\text { No breakdown by SES }\end{array}$ \\
\hline Factors of interest & $\begin{array}{l}\text { Study examines potential factors associated } \\
\text { with the differences in healthcare utilisation } \\
\text { between socioeconomic groups }\end{array}$ & $\begin{array}{l}\text { Studies researching the effect of interventions } \\
\text { on differences in healthcare utilisation between } \\
\text { socioeconomic groups }\end{array}$ \\
\hline Publication date & - All dates are included & \\
\hline Study design & $\begin{array}{l}\text { Quantitative study } \\
\text { Reviews } \\
\text { - Mixed method studies }\end{array}$ & - Qualitative study \\
\hline Type of publication & $\begin{array}{l}\text { Journal articles } \\
\text { Eligible papers underlying reviews }\end{array}$ & $\begin{array}{l}\text { Grey literature } \\
\text { Other non-peer-reviewed publications }\end{array}$ \\
\hline
\end{tabular}

OECD, Organisation for Economic Co-operation and Development; SES, socioeconomic status. 
to seek preventive care might be different to curative care. Except for interventions or health policies aiming to reduce socioeconomic inequality in healthcare utilisation, no predefined restrictions are placed on the eligible factors to capture the entire spectrum. Furthermore, SES is interpreted in a broad sense, meaning that an indicator is regarded as an SES measure whenever the initial authors perceive it as such.

\section{Study population}

For socioeconomic measures to be reflective of an individual's SES, the population is restricted to the adult population ( $\geq 18$ years). Eligibility is restricted to OECD countries with UHC to increase comparability. Social gradients are evident in countries with universal access to care or broad social benefits. The focus of this research is on factors explaining any socioeconomic differences in countries that provide universal access and broad welfare programmes, which is operationalised as OECD countries with UHC. ${ }^{18}{ }^{19}$ In effect, this aggregates to all OECD member states, excluding the USA and Mexico. ${ }^{20}$ A list of OECD countries with universal health coverage is provided in the online supplemental files.

\section{Study characteristics}

We included peer-reviewed articles that quantify the effect of any factor on the relation between SES and healthcare utilisation. Additional qualitative information, for example, from review papers or mixed-method papers, will be incorporated into the narrative synthesis. Original articles that underlie relevant review papers will be included if they meet the selection criteria. Grey literature and literature written in a language other than English or Dutch are not considered. No restrictions are made based on the publication date.

\section{Search strategy}

The search strategy covers the key concepts of the research: SES, healthcare, utilisation, inequality and data analysis. All terms within a key concept are searched within title and abstract and combined using the Boolean operator OR. All key concepts are joined using AND. The glossary of terms for the search strategy is based on an iterative process in which relevant articles have been scanned for words and synonyms expressing the five key concepts of interest. Where possible, indexing terms and restrictions of the geographical area, language and type of publication have been incorporated in the search strategy. A complete overview of the search strategy in PubMed is provided in the online supplemental files. The search strategy was applied to the following databases on 9 March 2021: Embase, PubMed, Web of Science, Scopus, Econlit and PsycInfo. An update to the search will be performed towards the end of the study.

\section{Selection procedure}

Results from each database will be combined, and duplicates will be removed using Endnote. Thereafter, results will be imported to Rayyan for the selection process.
Title and abstract will be screened based on relevance and geographical area by at least two reviewers independently (IM, NS and a third researcher (to be determined)). The remaining articles will be screened in full-text by at least two reviewers independently (IM, NS and a third researcher (to be determined)) for the predefined criteria in table 1. Disagreements will be resolved by referring to the protocol and/or by consulting the third researcher and/or an independent researcher (EU). The degree of agreement between assessors will be captured by Cohen's Kappa statistic. A backward and forward snowball procedure will be applied to the articles that meet the selection criteria. Original articles underlying relevant review papers will be extracted and included if they meet the selection criteria. The selection procedure will be presented in a PRISMA flowchart for which a template is provided in the online supplemental files.

\section{Data extraction procedure}

Data will be extracted using a predetermined data extraction form in Formdesk. The data extraction form is based on reporting items set out in the PRISMA guidelines and modified for this study. Data extraction categories will include general study characteristics, aim/objectives of the study, sample characteristics, data characteristics, study design, study inclusion/exclusion criteria, factors, main outcomes, and strengths and limitations. If applicable, fields will have decision rules about coding data to avoid ambiguity and to aid consistent completion. Data extraction forms will be piloted on a sample of included studies (approximately $5 \%$ of the study sample) to ensure that all the relevant information is captured. One researcher extracts the data (IM); a second researcher independently checks the data extraction forms for accuracy and completeness (EU or NS).

\section{Quality appraisal}

A quality appraisal will be conducted to evaluate the risk of bias within the included studies. Hoy's risk of bias tool, ${ }^{21}$ which covers, among others, the population representativeness, sample selection, data collection procedure and prevalence period, will be used as the outset for the assessment. The risk-of-bias tool will be tailored specifically to this systematic review. Two independent raters will assess each study based on the items of the tool. For each item, the raters will judge the risk of bias as 'high risk' or 'low risk' and provide quotes or comments to support their decision. The item can be classified as 'unclear' in case of insufficient available information. An overall judgement on the risk of bias will also be assessed in the form of a summary item, which can be rated as 'low risk', 'moderate risk' or 'high risk'. Any disagreement between raters will be resolved by discussion or consulting a third rater. Synthesis will be stratified based on the ruling of low risk for key study design elements such as population representativeness. 


\section{Synthesis of results}

Given the broad nature of the research question, great variability is expected in SES indicators, type of healthcare, examined factors, populations/subgroups and methodology. Due to this clinical and methodological diversity, a meta-analysis of effect estimates may not be possible. ${ }^{22}$ If so, the studies' characteristics will be described in a numerical summary, and data will be synthesised narratively and discussed thematically. As some findings will likely be bounded to a specific context, results will be distinguished according to generalisability. Furthermore, factors examined in the quantitative research and the direction of the association will be reported by type of healthcare service. Significant factors will be categorised and compared with the dimensions and abilities identified by Levesque $e t$ al. ${ }^{9}$ In case of sufficiently comparable outcomes, statistical synthesis methods will be applied, such as vote counting ('is there any evidence of an effect?') or a summary of effect estimates ('what is the range and distribution of observed effects?') ${ }^{23}$ If of added value, an overview of the findings may be presented in a table or figure. Any qualitative evidence that comes along with the eligible studies will be incorporated into the narrative synthesis. The robustness of synthesis is assessed by reflecting critically on the synthesis process.

\section{Study limitations}

The main limitation of the proposed systematic review is related to the expected heterogeneity in study characteristics and outcome measures of the included studies, limiting the comparability and statistical analysis. For analogy, this study excludes preventive care, quality of care and health status as outcomes. The scope is further restricted to OECD countries with UHC to reduce the role of contextual differences, for example, financial accessibility of care. Another limitation of this study could be that the data extraction procedure will be performed by one researcher. In order to avoid errors, the data extraction will be checked independently by another researcher.

\section{ETHICS AND DISSEMINATION}

No primary data are collected for this systematic review. As we will only make use of secondary data, ethics approval is not required.

\section{Dissemination}

To enhance the study design and eliminate any study biases, this systematic review protocol is published in advance. We intend to publish our findings in an international peer-reviewed journal.

\section{Patient and public involvement statement \\ Patients and the public are not involved in this research.}

Contributors The initial manuscript was written by IM. EU, JP and NS revised and commented on draft versions of the manuscript. The search strategy was developed by IM and NS and further refined by EU and JP. All authors contributed to the determination of the study conception, design and eligibility criteria. All authors read and approved the final manuscript.

Funding This study is funded by the Strategic Program project 'SOCIETAL' of the Dutch National Institute for Public Health and the Environment (RIVM).

Competing interests None declared.

Patient consent for publication Not applicable.

Provenance and peer review Not commissioned; externally peer reviewed.

Supplemental material This content has been supplied by the author(s). It has not been vetted by BMJ Publishing Group Limited (BMJ) and may not have been peer-reviewed. Any opinions or recommendations discussed are solely those of the author(s) and are not endorsed by BMJ. BMJ disclaims all liability and responsibility arising from any reliance placed on the content. Where the content includes any translated material, BMJ does not warrant the accuracy and reliability of the translations (including but not limited to local regulations, clinical guidelines, terminology, drug names and drug dosages), and is not responsible for any error and/or omissions arising from translation and adaptation or otherwise.

Open access This is an open access article distributed in accordance with the Creative Commons Attribution Non Commercial (CC BY-NC 4.0) license, which permits others to distribute, remix, adapt, build upon this work non-commercially, and license their derivative works on different terms, provided the original work is properly cited, appropriate credit is given, any changes made indicated, and the use is non-commercial. See: http://creativecommons.org/licenses/by-nc/4.0/.

ORCID iD

Iris Meulman http://orcid.org/0000-0002-9916-1810

\section{REFERENCES}

1 van Doorslaer E, Wagstaff A, van der Burg H, et al. Equity in the delivery of health care in Europe and the US. J Health Econ 2000;19:553-83.

2 Lemstra M, Mackenbach J, Neudorf C, et al. High health care utilization and costs associated with lower socio-economic status: results from a linked dataset. Can J Public Health 2009;100:180-3.

3 Stirbu I, Kunst AE, Mielck A, et al. Inequalities in utilisation of general practitioner and specialist services in 9 European countries. BMC Health Serv Res 2011;11:288.

4 Loef B, Meulman I, Herber G-CM, et al. Socioeconomic differences in healthcare expenditure and utilization in the Netherlands. BMC Health Serv Res 2021;21:643.

5 Kunst A, Meerding W, Varenik N. Sociale verschillen in zorggebruik en zorgkosten in Nederland 2003. Zorg voor euro's-5 2007.

6 Fjær EL, Balaj M, Stornes P, et al. Exploring the differences in general practitioner and health care specialist utilization according to education, occupation, income and social networks across Europe: findings from the European social survey (2014) special module on the social determinants of health. Eur J Public Health 2017;27:73-81.

7 Lueckmann SL, Hoebel J, Roick J, et al. Socioeconomic inequalities in primary-care and specialist physician visits: a systematic review. Int J Equity Health 2021;20:58.

8 Van der Heyden JHA, Demarest S, Tafforeau J, et al. Socio-economic differences in the utilisation of health services in Belgium. Health Policy 2003;65:153-65.

9 Levesque J-F, Harris MF, Russell G. Patient-centred access to health care: conceptualising access at the interface of health systems and populations. Int J Equity Health 2013;12:18.

10 Droomers M, Westert GP. Do lower socioeconomic groups use more health services, because they suffer from more illnesses? Eur $J$ Public Health 2004;14:311-3.

11 Agerholm J, Bruce D, Ponce de Leon A, De Leon AP, et al. Socioeconomic differences in healthcare utilization, with and without adjustment for need: an example from Stockholm, Sweden. Scand J Public Health 2013;41:318-25.

12 Merlo J, Gerdtham U-G, Lynch J, et al. Social inequalities in healthdo they diminish with age? Revisiting the question in Sweden 1999. Int J Equity Health 2003;2:2.

13 Jansen T, Rademakers J, Waverijn G, et al. The role of health literacy in explaining the association between educational attainment and the use of out-of-hours primary care services in chronically ill people: a survey study. BMC Health Serv Res 2018;18:394.

14 Mondor L, Watson T, Kornas K, et al. Direct and indirect pathways between low income status and becoming a high-cost health care user in Ontario, Canada: a mediation analysis of health risk behaviors. Ann Epidemiol 2020;51:28-34. 
15 Chernew M, Gibson TB, Yu-Isenberg K. Effects of increased patient cost sharing on socioeconomic disparities in health care. $J$ Gen Intern Med 2008;23:1131-6.

16 Moher D, Liberati A, Tetzlaff J, et al. Preferred reporting items for systematic reviews and meta-analyses: the PRISMA statement. $B M J$ 2009;339:b2535.

17 Page MJ, McKenzie JE, Bossuyt PM, et al. The PRISMA 2020 statement: an updated guideline for reporting systematic reviews. BMJ 2021;372:n71.

18 Organisation for Economic Co-operation and Development. OECD member countries and partners 2021. Available: https://www.oecd. org/about/members-and-partners/\#
19 Tikkanen R, Osborn R, Mossialos E. International health care system profiles, 2020. Available: https://www.commonwealthfund.org/ international-health-policy-center/system-profiles

20 OECD. Society at a glance 2014. Paris: OECD Publishing, 2014: 130-1.

21 Hoy D, Brooks $P$, Woolf $A$, et al. Assessing risk of bias in prevalence studies: modification of an existing tool and evidence of interrater agreement. J Clin Epidemiol 2012;65:934-9.

22 McKenzie J, Brennan S. Chapter 12: synthesizing and presenting findings using other methods. In: Cochrane handbook for systematic reviews of interventions. Cochrane, 2021.

23 McKenzie J, Brennan S, Ryan R. Chapter 9: summarizing study characteristics and preparing for synthesis. In: Cochrane handbook for systematic reviews of interventions. Cochrane, 2021. 